\title{
Driven vortices in type-II superconductors: A muon spin rotation study
}

\author{
D. Charalambous, * E. M. Forgan, S. Ramos, S. P. Brown, R. J. Lycett, and D. H. Ucko ${ }^{\dagger}$ \\ School of Physics \& Astronomy, University of Birmingham, Birmingham B15 2TT, United Kingdom \\ A. J. Drew ${ }^{\ddagger}$ and S. L. Lee \\ School of Physics \& Astronomy, University of St. Andrews, St. Andrews, Fife KY16 9SS, United Kingdom \\ D. Fort \\ School of Metallurgy \& Materials, University of Birmingham, Birmingham B15 2TT, United Kingdom
}

A. Amato and U. Zimmerman

Paul Scherrer Institut, CH-5232 Villigen PSI, Switzerland

(Received 8 September 2005; revised manuscript received 7 February 2006; published 27 March 2006)

\begin{abstract}
We present a detailed study of vortex motion in a type-II superconductor using the muon spin rotation $(\mu \mathrm{SR})$ technique. The vortices were set in motion by an alternating transport current. By adjusting the frequency and amplitude of the driving force so that vortices "visible" to the implanted muons did not cross the sample boundaries, a $\mu$ SR line shape was obtained corresponding to almost perfectly ordered vortex motion. We also observed sidebands to the $\mu \mathrm{SR}$ line shape which correspond to the frequency at which vortices pass the implanted muon, and allow a direct measurement of the vortex velocity. Both these features of the line shape confirm numerical predictions reported in a previous study [see Charalambous et al., Phys. Rev. B 66, 054506 (2002)].
\end{abstract}

DOI: 10.1103/PhysRevB.73.104514

PACS number(s): 74.25.Qt, 74.25.Fy, 74.70.Ad, 76.75.+i

\section{INTRODUCTION}

It is well known that when a transport current is applied to a type-II superconductor in the mixed state, individual vortex lines experience a Lorentz force $\boldsymbol{F}_{\mathrm{L}}$ per unit volume given by ${ }^{1}$

$$
\boldsymbol{F}_{\mathrm{L}}=\boldsymbol{J} \wedge \boldsymbol{B},
$$

where $\boldsymbol{J}$ is the applied current density and $\boldsymbol{B}$ is the magnetic induction in the superconductor. The vortices are normally pinned by quenched disorder (e.g., material defects such as grain boundaries, dislocations, second phase particles, roughness of the sample surfaces, etc.) but when $\boldsymbol{F}_{\mathrm{L}}$ exceeds the pinning force, the vortex lines are set in motion with an average velocity $\boldsymbol{v}$. Moving vortices give rise to an electric field $^{2}$

$$
\boldsymbol{E}=\boldsymbol{v} \wedge \boldsymbol{B},
$$

and hence energy dissipation occurs. This is due to the relaxation of the order parameter as well as due to normal currents in the vortex core. ${ }^{3-5}$

As an intriguing example of nonequilibrium physics, systems of moving vortices have attracted much attention from both experimentalists and theorists. ${ }^{6-13}$ Their dynamic properties are governed by an interplay between disorder, thermal fluctuations, and driving force, as well as applied magnetic field, temperature, and dimensionality. Furthermore, from a technological point of view, a detailed understanding of vortex motion is crucial for the design and manufacture of superconductors to be used in various applications, since vortex motion-induced energy dissipation is one of the main factors that limits the range of applicability of these materials. $^{14}$

It is now well established that the moving vortex system is more ordered than it is in the pinned state: theoretical and computational investigations predicted a variety of dynamic phases, ${ }^{6-9,15}$ all of which are consistent with the moving vortex system exhibiting orientational ordering compared to the static, pinned case. Recent small-angle neutron scattering (SANS) experiments on low-temperature $\mathrm{Pb}$-alloy superconductors $^{10-12,16}$ show the ordering of the vortex system in the flux flow state. The flux lines give a diffraction pattern similar to that expected from a vortex lattice. This manifests itself as six Bragg peaks in the structure factor, arranged in a hexagonal pattern. A vortex nearest-neighbor direction tends to lie parallel to the direction of vortex motion; however, the moving vortex system does not form a perfectly aligned crystal, as can be seen by careful measurement of the transverse width of the Bragg peaks.

In this paper is presented a detailed experimental and theoretical investigation of vortex motion in type-II superconductors using muon spin rotation $(\mu \mathrm{SR})$. In a previous study, ${ }^{17}$ vortex motion was induced by the application of a pulsed, unidirectional driving force and it was found that the moving vortex system exhibited a significant amount of disorder. In the present work, we have studied the effects of an alternating driving force, and we find that this tends to cause a greater degree of dynamic ordering of the vortex lines. The ordering shows a dependence on the frequency and amplitude of the alternating force, which suggests that a significant part of the disordering observed in our previous unidirectional study resulted from vortex lines crossing the sample boundaries, and the disorder is therefore not an intrinsic property of the moving vortex system. It is also possible that 
the earlier results partly reflect the very high frequencies represented by the pulsed driving force.

The experimental method and results are presented in Sec. II, followed by a detailed discussion in Sec. III. The conclusions from this work are presented in Sec. IV. An analytical discussion of $\mu \mathrm{SR}$ line shapes from moving vortex lattices is given in the Appendix.

\section{EXPERIMENTAL}

\section{A. Experimental method}

The $\mu \mathrm{SR}$ experiments were carried out using the General Purpose Surface muon facility (GPS) at the Paul Scherrer Institut, Switzerland. The GPS spectrometer has available a muon spin rotator which allows the muon spin polarization to be rotated to about $60^{\circ}$ from the momentum direction. ${ }^{18}$ The muons are implanted individually in a sample placed in a magnetic field parallel to their incoming momentum. The perpendicular component of the spin of each muon precesses about the field axis (which will be referred to as the $z$ direction). The frequency of precession is given by $\gamma_{\mu} B_{z}$, where $B_{z}$ is the local value of the magnetic field at the muon implantation site, and $\gamma_{\mu}$ is the gyromagnetic ratio of the muon $\left(\gamma_{\mu} / 2 \pi \approx 135.54 \mathrm{MHz} \mathrm{T}^{-1}\right)$. When a muon decays (with a lifetime $\tau_{\mu}$ of $2.197 \mu \mathrm{s}$ ), the decay positron is emitted preferentially along the muon spin direction. Scintillators around the sample detect the positrons; the counts from typically $10^{7}$ muons are recorded in histograms as a function of time after muon implantation. From such data, the time evolution of the average muon polarization can be deduced, and hence the distribution of local values of magnetic field.

In our experiments, the complex muon polarization $P(t)$ $=P_{x}+i P_{y}$ was obtained by utilizing a set of positron detectors around the sample in the region of the $x y$ plane, with the magnetic field applied in the $z$ direction. The magnetic field probability density function $p(B)$, which gives rise to the precession signal in the $x y$ plane, was obtained from the $\mu \mathrm{SR}$ data by employing a maximum-entropy-based inversion algorithm. ${ }^{19-22}$ The function $p(B)$ is related to $P(t)$ according to the equations ${ }^{17}$

$$
\begin{gathered}
p(B)=\operatorname{Re}\left\{\frac{\gamma_{\mu}}{\pi P(0)} \int_{0}^{\infty} P(t) e^{-i \gamma_{\mu} B t} d t\right\}, \\
P(t)=P(0)\left\langle\exp \left(i \gamma_{\mu} \int_{0}^{t} B(\boldsymbol{r}-\boldsymbol{v} \tau) d \tau\right)\right\rangle_{\boldsymbol{r}} .
\end{gathered}
$$

The brackets $\langle\cdots\rangle_{r}$ denote an average over all muon implantation sites with position vector $\boldsymbol{r}$, and $\boldsymbol{v}$ is the velocity of the vortex lattice.

The sample used in the measurements was a plateletshaped, polycrystalline $\mathrm{Pb}-\mathrm{In}$ alloy, measuring $15 \times 15$ $\times 0.5 \mathrm{~mm}^{3}$ and prepared in the same way as in our previous study ${ }^{17}$ It was placed with its flat surface perpendicular to the applied magnetic field and the incoming muon beam $(z$ direction). The current leads consisted of copper strips coated with $\mathrm{Pb}-\mathrm{Sn}$ solder. At the temperatures and magnetic fields used in our experiments, the solder was superconduct-

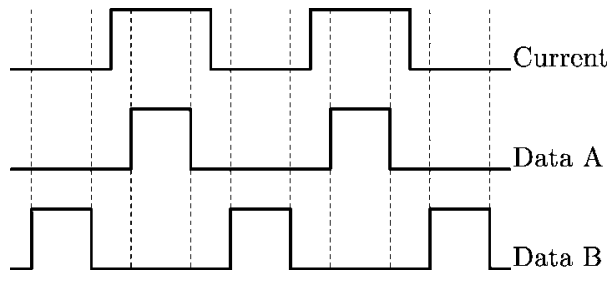

FIG. 1. The current pulse sequence applied to the sample relative to the data acquisition routing signals.

ing and the leads had a critical current much larger than that of the sample. The current leads were soldered along two opposite edges of the sample, providing a transport current in the horizontal $(y)$ direction (that is, perpendicular to the incoming muons and applied field). This geometry gives rise to vortex motion in the vertical $(x)$ direction. A mylar shield, coated with an $\sim 1 \mathrm{~mm}$ thick layer of antiferromagnetic $\mathrm{Fe}_{2} \mathrm{O}_{3}$, was placed symmetrically in front of the sample, exposing the central area $\left(10 \times 10 \mathrm{~mm}^{2}\right)$ to the incoming muons. The strong atomic magnetic fields in $\mathrm{Fe}_{2} \mathrm{O}_{3}$ ensure that any muons stopping outside the central region of the specimen do not contribute to the precession signal.

The superconducting specimen was placed inside a continuous flow cryostat, which provided efficient helium gas flow cooling of both the sample and the superconducting transport current leads connected to it. The sample was cooled in an applied magnetic field to establish the equilibrium density of vortex lines in the specimen before each series of measurements.

Initially, a unidirectional pulsed transport current was applied to the sample. As illustrated schematically in Fig. 1, two routing signals (labeled "Data A" and "Data B") were also created. They were used to gate the data acquisition electronics so that measurements could be taken both with and without a current flowing through the sample and stored in separate histograms. The current pulse sequence had 1:1 mark-space ratio, with data acquisition gated to the central $90 \%$ of each half-period. This gating ensured that any transients in the current pulse had died away and vortex motion had reached steady state, before data acquisition began, and that data-taking ended well before the current changed again. The current was provided by a dc superconducting magnet power supply, the output of which was passed either through the sample or through a dummy load. This was achieved by using a simple field-effect transistor (FET) switching circuit, which was driven by a programmable function generator. The current was switched typically at tens of $\mathrm{Hz}$, so that if heating by the current were important, the sample would remain warm during the current-off period. Data taken during the current-off period were used to confirm that the observed effects on the $\mu \mathrm{SR}$ line shape were due to vortex motion and not due to heating.

A different FET switching circuit was subsequently used to supply a square-wave alternating current to the sample. The "Data A" (B) signals of Fig. 1 gated the acquisition electronics to collect data during positive (negative) current flowing through the superconductor. 


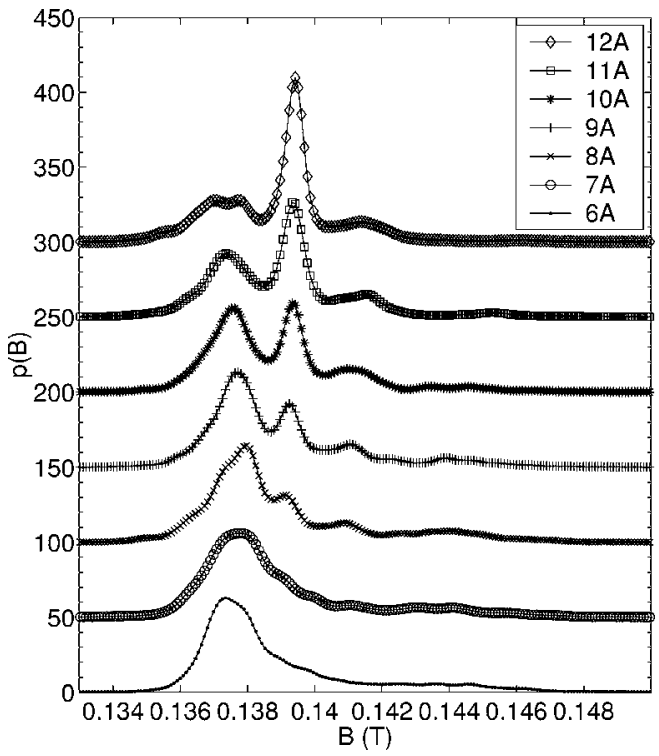

FIG. 2. $\mu$ SR line shapes obtained at $2 \mathrm{~K}$ and $\mu_{0} H_{a}=0.14 \mathrm{~T}$, for a range of unidirectional transport currents applied at $10 \mathrm{~Hz}$. For clarity, each curve is shifted vertically by 50 units relative to the previous one.

\section{B. Experimental results}

Figure 2 shows the $\mu \mathrm{SR}$ spectra obtained at a temperature of $2 \mathrm{~K}$ and an applied field $\mu_{0} H_{a}=0.14 \mathrm{~T}$, for a range of unidirectional pulsed transport currents.

It can be seen that $p(B)$ is strongly modified from the characteristic form for a static triangular vortex lattice. As the current is increased, a peak develops at approximately $0.139 \mathrm{~T}$, which corresponds to the average magnetic induction in the sample; for very large currents, $p(B)$ is said to be motionally narrowed, similar to the results in Ref. 17.

The effect of current flow on $p(B)$ cannot be due to heating of the sample, since the $p(B)$ obtained between the current pulses, shown in Fig. 3, is almost identical to that shown in Fig. 2 for a very small (below critical) applied current.

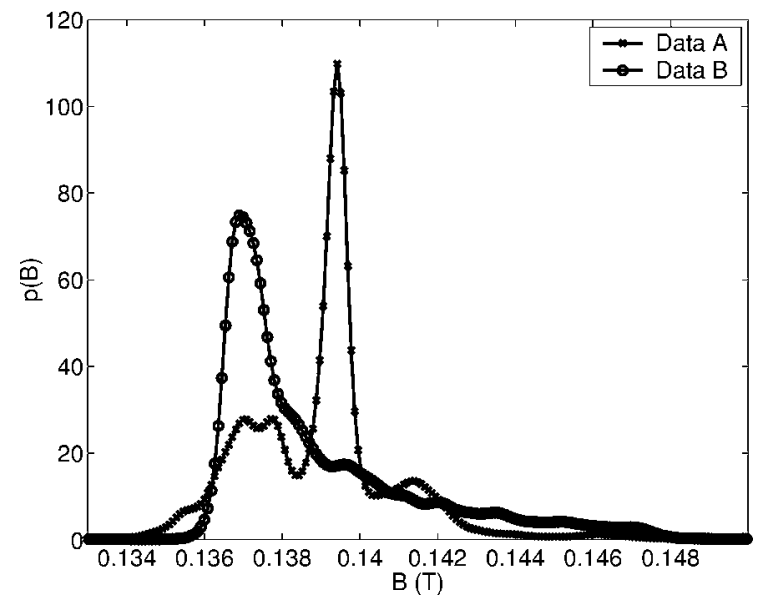

FIG. 3. The 12 A line shape shown in Fig. 2 (labeled Data A) plotted along with the line shape corresponding to data collected between the current pulses (Data B).

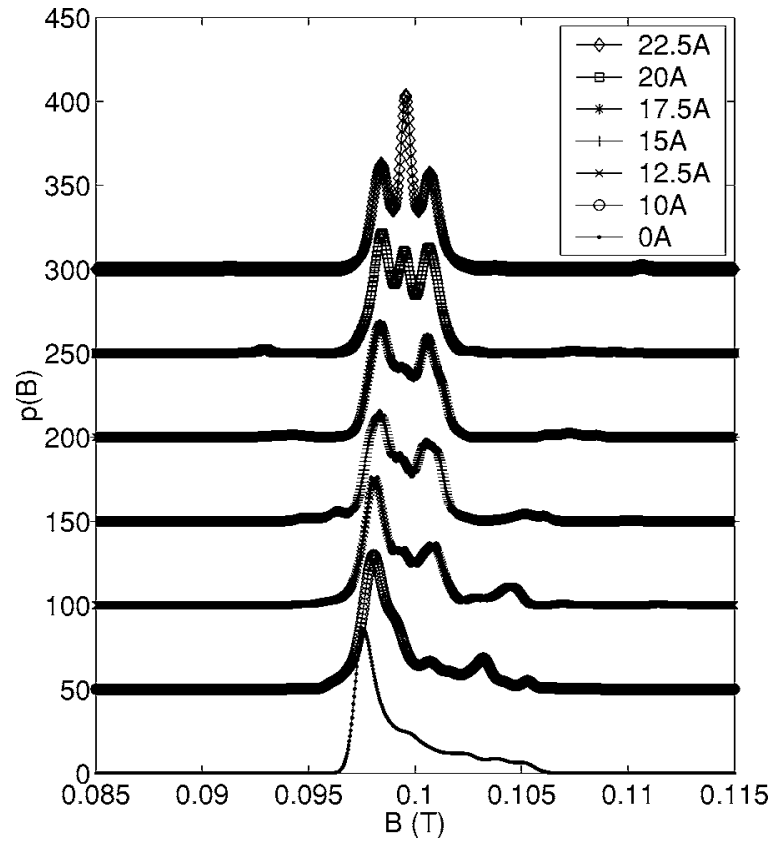

FIG. 4. $\mu$ SR line shapes for a range of alternating transport current amplitudes, obtained for $\mu_{0} H_{a}=0.1 \mathrm{~T}$ at $4.2 \mathrm{~K}$. The frequency of the current pulses was $25.9 \mathrm{~Hz}$.

The dramatic difference between "current on" and "current off" results in Fig. 3 must therefore be due to vortex motion.

The $\mu$ SR spectra obtained when an alternating squarewave current was applied to the sample are shown in Fig. 4. These were obtained at $4.2 \mathrm{~K}$, with $\mu_{0} H_{a}=0.1 \mathrm{~T}$ and with current applied at a frequency of $25.9 \mathrm{~Hz}$. (The data for both current flow directions were identical within experimental error, so they were analyzed together to give these results.) For low driving current amplitude, these line shapes are significantly different from those in Fig. 2: full motional narrowing is not observed for currents up to $15 \mathrm{~A}$. Instead, the line shapes exhibit a characteristic double-peak structure, similar to that predicted theoretically in Ref. 17 for the case of ordered vortex motion along a principal vortex lattice direction.

An important feature of the spectra in Fig. 4 is the existence of small sidebands on either side of the main $p(B)$ distribution (see also Fig. 5). These sidebands also exist in the line shapes of Fig. 2, but they are broader and less pronounced. In order to make the sidebands more visible, the line shapes of Fig. 4 are also plotted with the vertical scale increased in Fig. 5.

It should finally be noted that all these measurements were taken at much higher fields than those presented in Ref. 17. This is mainly due to the finite time-width of the muon pulses used in Ref. 17; this reduces the amplitude of the precession signal at high frequencies, and hence limits the field value that can be used to observe muon spin rotation. In the present work, the higher applied magnetic field has the advantage that the flux flow state can be attained at relatively low transport currents. 


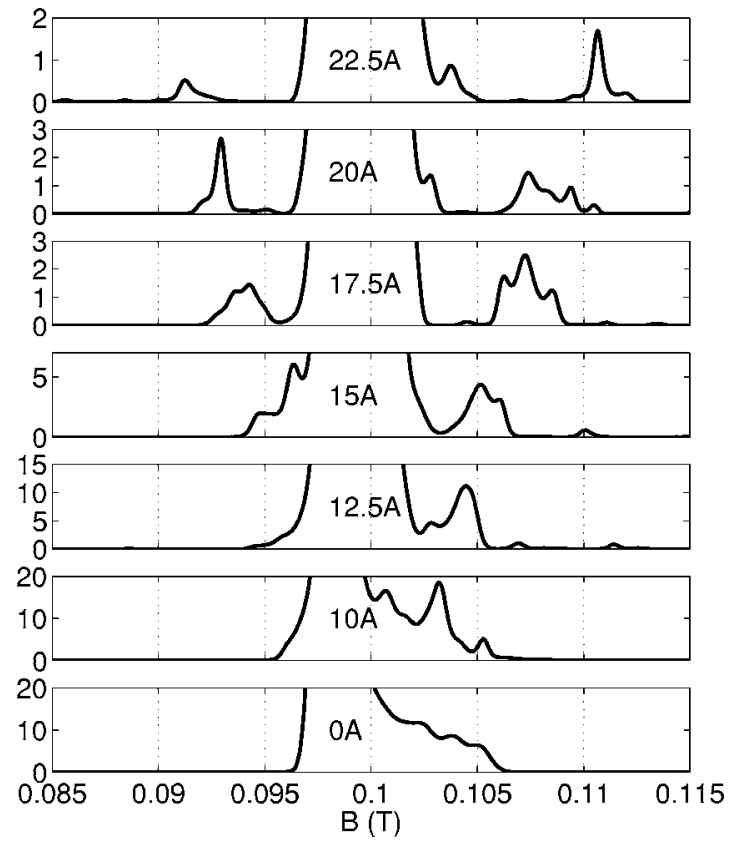

FIG. 5. The $\mu$ SR line shapes of Fig. 4, with the vertical scale altered in order to make small sidebands more visible.

\section{DISCUSSION}

\section{A. Unidirectional driving force}

The line shapes obtained for unidirectional pulsed current (see Fig. 2) are consistent with those obtained by Ref. 17 However, the present results refer to a much higher applied field and simpler sample/field geometry. (That is, the sample was placed at right angles to the applied field as opposed to the $45^{\circ}$ geometry, which was necessarily employed in Ref. 17.)

It is clear that when the vortex lattice moves, the timeaveraged magnetic field at any point is more spatially uniform than that for a static vortex lattice. Hence some motional narrowing of the field distribution $p(B)$ is to be expected at large driving currents. However, the numerical calculations presented in Ref. 17 demonstrate that aligned motion of the vortex lattice does not cause complete motional narrowing. On the other hand, when the vortex lattice moves with a range of orientations, the field at the muons motionally averages to a single peak at large velocities. The field distributions plotted in Fig. 2 are in good agreement with the nonordered simulations in Ref. 17, implying that there is a significant amount of disorder in the orientation of the moving vortex system. It is important to note that the moving vortex system is not completely disordered. As shown in Ref. 17, the vortex system exhibits some degree of orientational order and moves, on average, along a principal vortex lattice direction. This was confirmed by SANS measurements, where the structure factor $S(\boldsymbol{q})$ was found to be anisotropic, consisting of six Bragg peaks arranged in a hexagonal pattern. However, the transverse width of these peaks was much larger than the radial width, implying a range of vortex lattice orientations.

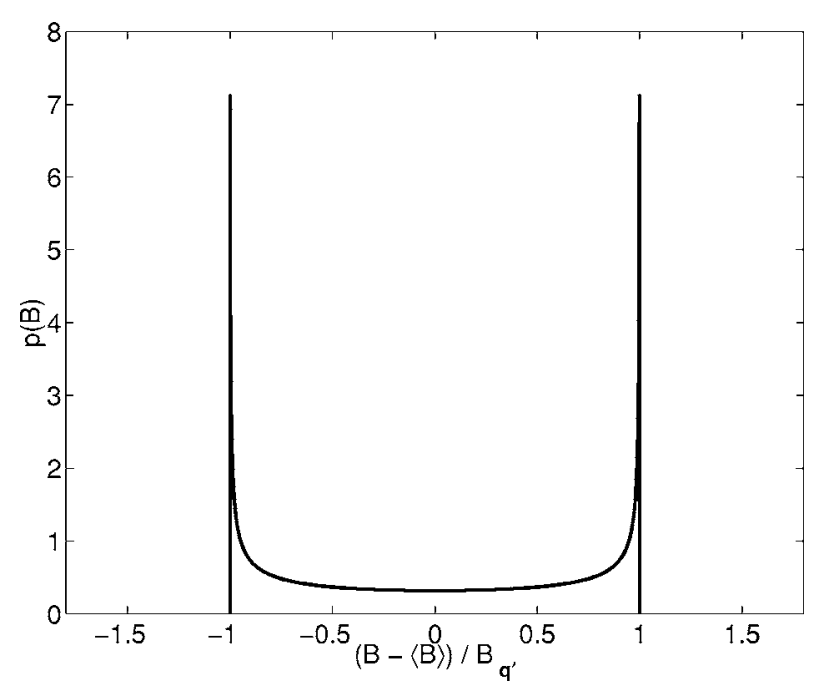

FIG. 6. Idealized magnetic field probability distribution corresponding to vortex motion along a Bragg plane, for $|\boldsymbol{v}| \rightarrow \infty$ and only one reciprocal lattice vector contributing to the field distribution (see the Appendix for details).

\section{B. Spectral shapes}

A detailed analytical discussion of the expected line shapes is given in the Appendix. There are two distinct mathematical cases to consider, depending on the orientation of the vortex lattice velocity relative to any plane running from vortex to vortex through the lattice. The simplest of such planes runs through nearest-neighboring vortices in the lattice (and is the favored direction of motion for the lattice). However, there are other planes running through more distant neighbors. These planes all correspond to Bragg planes of vortices which give rise to the diffraction pattern of the vortex lattice. We first consider the "unsymmetrical" case when the vortex velocity does not lie in any Bragg plane of the vortex lattice. In this case, we demonstrate explicitly a complete motional narrowing of the line shape at large vortex velocities. Conversely, if the vortex lattice moves in a direction exactly parallel to a Bragg plane, then at large vortex velocities a characteristic two-peaked line shape is seen, with a shape described by Eq. (A15), which is plotted in Fig. 6. As discussed in Ref. 17, this line shape arises because the time averaged field due to a rapidly moving orientationally ordered vortex lattice varies only in the direction perpendicular to the vortex velocity. The field variation in this direction is approximately sinusoidal, with a maximum field value along the lines traversed by the vortices and a minimum field value between these lines. The two peaks in $p(B)$ at large velocities are at the values of the maximum and minimum time-averaged fields.

At finite vortex lattice velocities, our analytical calculations predict the appearance of small "sideband" peaks, which are separated from the main peaks by multiples of the vortex lattice "washboard frequency" $\nu_{\mathrm{wb}}$, which is the frequency at which vortex lattice unit cells move past an implanted muon. Such sidebands are visible in the experimental data shown in Figs. 2, 4, and 5 and the numerical simulations in Ref. 17. The existence of such features may be understood 
by considering the precession signal of an implanted muon as unit cells of the vortex lattice sweep past (at a frequency much lower than the muon precession frequency). While a field maximum of the vortex lattice is passing over the muon, it will precess faster than average; conversely, the muon will precess slower than average while a field minimum lies over it. Thus the precession signal from any implanted muon may be thought of as a frequency-modulated signal. As is well known in telecommunications theory, such frequency modulation gives rise to sideband peaks in the frequency spectrum, spaced from the average frequency by multiples of the modulation frequency (see, e.g., Ref. 23). The frequency spectrum of the moving vortex lattice as a whole will be determined by an average over the unit cell. There will be dominant contributions from regions where the time-average field is varying little with position, i.e., near the lines where the time-average field is maximum and minimum. These regions give rise to the two peaks in Fig. 6. Telecommunication theory also shows that the strength of the sidebands increases with the amplitude of frequency modulation. Along a line where the vortex centers pass, the field value varies between the vortex core field and the saddle point field halfway between cores. Along a line half a unit cell away, the field varies from the minimum value to the saddle point value. The amplitude of frequency modulation is larger in the former case. This region gives rise to the higher-field peak of the pair seen at large velocities. Hence, the stronger sidebands will be satellites of the higher-field peak.

The sidebands are of interest because they are a direct consequence and measure of the local vortex lattice velocity. Let $B_{\mathrm{wb}}$ be the distance of the first sideband peak from the rightmost main peak of the $p(B)$ spectrum. The vortex lattice velocity $v$ is related to the washboard frequency by

$$
\frac{v}{a_{0}}=\nu_{\mathrm{wb}}=\frac{\gamma_{\mu}}{2 \pi} B_{\mathrm{wb}},
$$

where $a_{0}$ is the equilibrium vortex line spacing. However, from Eq. (2), the flux flow velocity $v$ can also be expressed in terms of the value of the voltage, $V_{\mathrm{ff}}$, across the sample due to flux flow,

$$
v=\frac{V_{\mathrm{ff}} / \ell}{B},
$$

where $\ell$ is the separation of the voltage contacts (equal to $15 \mathrm{~mm}$ in these measurements) and $B$ is the average magnetic induction. This allows a direct comparison of microscopic and macroscopic measurements of this velocity.

$B_{\mathrm{wb}}$ was estimated from the line shapes of Fig. 5 and converted to a flux flow velocity according to Eq. (5). This $\mu$ SR-determined flux flow velocity is plotted in Fig. 7 along with that determined using a conventional voltmeter connected across the sample. (The voltage measurements were carried out at a factor $\sim 100$ lower frequency to allow for the dc voltmeter settling time.)

Figure 7 indicates that there is reasonable agreement between transport current-voltage measurements and $\mu \mathrm{SR}$, bearing in mind that, for a given applied current, there exists a range of values of $v$ as well as a range of vortex lattice

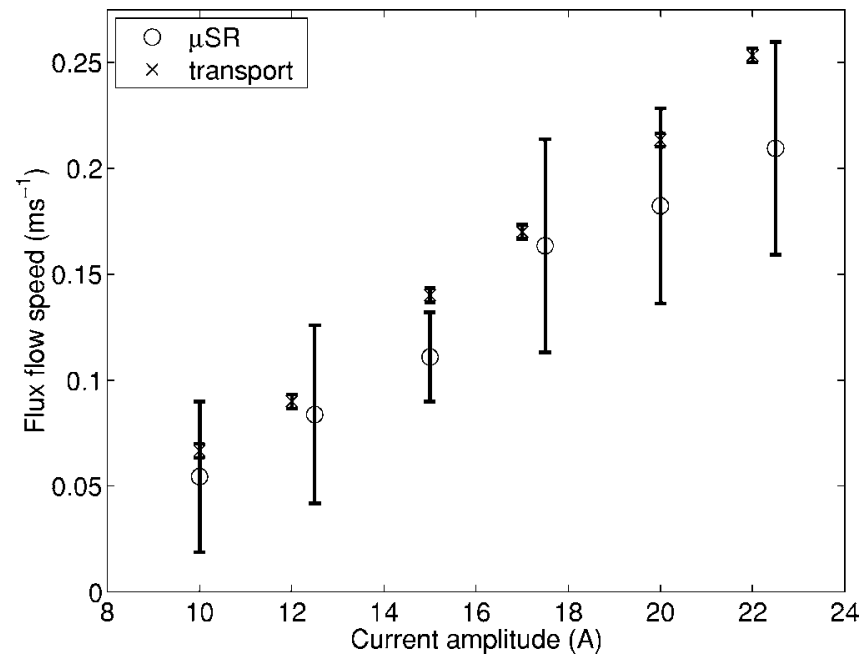

FIG. 7. Flux flow-current characteristics, obtained from $\mu \mathrm{SR}$ and transport measurements for $\mu_{0} H_{a}=0.1 \mathrm{~T}$ and temperature of $4.2 \mathrm{~K}$. These both extrapolate to a zero flux flow velocity at a critical current $\sim 6 \mathrm{~A}$.

orientations. Both of these departures from ideal behavior will broaden the sidebands and make the calculation of $v / a_{0}$ difficult and relatively inaccurate. Nevertheless, the agreement between transport and $\mu \mathrm{SR}$ measurements further confirms the validity of the model used to account for our results.

Analogous measurements were carried out on sliding charge-density waves (CDWs) by Jánossy et al. ${ }^{24}$ They induced a drift velocity of the $\mathrm{CDW}$ in $\mathrm{Rb}_{0.3} \mathrm{MoO}_{3}$ by passing a current, and measured the voltage and voltage noise and NMR spectrum as a function of the CDW current. Weak sidebands were observed in the NMR spectra, indicating a periodic temporal modulation of the local field at a nucleus, due to the moving CDW. The position of these sidebands was consistent with that expected from other expressions for the CDW drift velocity.

\section{Alternating driving force}

If a square wave alternating transport current $I$ of frequency $f$ is applied to the sample, the vortex lines will experience an alternating force, causing them to move forwards and backwards at a frequency $f$, provided that the amplitude of the current exceeds the critical current, $I_{c}$. In the experiments reported here, the mark-space ratio of the current pulses is $1: 1$, and it is easy to see that the vortex lines located more than a distance $d$ from the edges of the sample, where

$$
d=\frac{v}{2 f},
$$

will not cross the sample boundaries during a current cycle. Since the $\mathrm{Fe}_{2} \mathrm{O}_{3}$-coated mylar window symmetrically covers a "strip" of width $2.5 \mathrm{~mm}$ around the sample, then if $v /(2 f)<2.5 \mathrm{~mm}$ the vortex lines "visible" to the implanted muons will not have passed through the sample boundaries. For $f=25.9 \mathrm{~Hz}, B=0.1 \mathrm{~T}, \ell=15 \mathrm{~mm}$, and $d=2.5 \mathrm{~mm}$, this 
condition is satisfied for any flux flow voltage $V_{\text {ff }} \lesssim 2$ $\times 10^{-4} \mathrm{~V}$. This corresponds to a transport current amplitude of approximately $15 \mathrm{~A}$, as can be calculated from Fig. 7. For higher applied current amplitudes, a certain fraction of the vortex lines "visible" to the implanted muons will have passed through the sample edges. For instance, for a current of $17 \mathrm{~A}, V_{\mathrm{ff}} \approx 2.5 \times 10^{-4} \mathrm{~V}$. Hence vortex lines move, on average, a distance of $3.2 \mathrm{~mm}$ during a current cycle, corresponding to $0.7 \mathrm{~mm}$ inside the mylar window. The visible fraction is thus $2 \times 0.7 \times 10 /\left(10^{2}\right)=0.14$. In other words, $14 \%$ of the vortex lines within the mylar window have interacted with the sample boundaries. For a current of $20 \mathrm{~A}$, this fraction is $32 \%$ and $46 \%$ for $22.5 \mathrm{~A}$. These results are consistent with the line shapes of Fig. 4: when the fraction of vortex lines interacting with the sample edges (and visible to the implanted muons) is small (less than about 15\%), the $p(B)$ has a characteristic double-peak structure, which corresponds to well-ordered vortex motion along a principal vortex lattice direction. ${ }^{17}$ When a larger fraction of vortex lines within the mylar window have interacted with the sample boundaries, motional narrowing is clearly observed.

For a given vortex speed, the fraction of vortices interacting with the sample boundaries could be decreased by increasing the sample size or increasing the driving frequency. The former option was not available: our sample was as large as could conveniently fit the cryostat. The latter option was attempted but resulted in greater vortex disorder. The exact causes of this are a subject of further investigation. It is probable that the disorder is associated with the fact that the flux lines are slightly curved by the influence of the current, and the curvature changes sign every time the current reverses. This effect probably becomes particularly important when the frequency is large enough that the ac skin depth in the sample becomes comparable with its thickness. If this is the case, much thinner samples would not show the vortex lattice disordering at high frequencies. It is of interest to note that in our earlier measurements, ${ }^{17}$ the vortex motion was caused by very high frequency (unidirectional) pulses, and was always observed to be disordered.

Our observations of the effects of sample edges on vortex motion are consistent with those of Xiao et al..$^{13}$ by carrying out careful current-voltage $(I-V)$ measurements on $2 \mathrm{H}-$ $\mathrm{NbSe}_{2}$ crystals, they found that the nonlinear features in the measured $I-V$ characteristics are due to edge currents, which in turn depend on vortices crossing the sample boundaries through a current-dependent surface barrier. On the other hand, the bulk $I-V$ characteristics were found to be linear, corresponding to vortex motion in the bulk of the sample.

We now make a more detailed comparison between a particular example of our results and a simulation of the same situation. To discuss the latter, Charalambous et al. ${ }^{17}$ introduced a dimensionless measure of vortex velocity, $R$, given by

$$
R=\frac{2 \pi|\boldsymbol{v}|}{\gamma_{\mu} a_{0} \Delta B},
$$

where $\Delta B=\left(B_{\max }-B_{\min }\right)$ is the width of $p(B)$ corresponding to a stationary vortex system (a similar dimensionless quan-

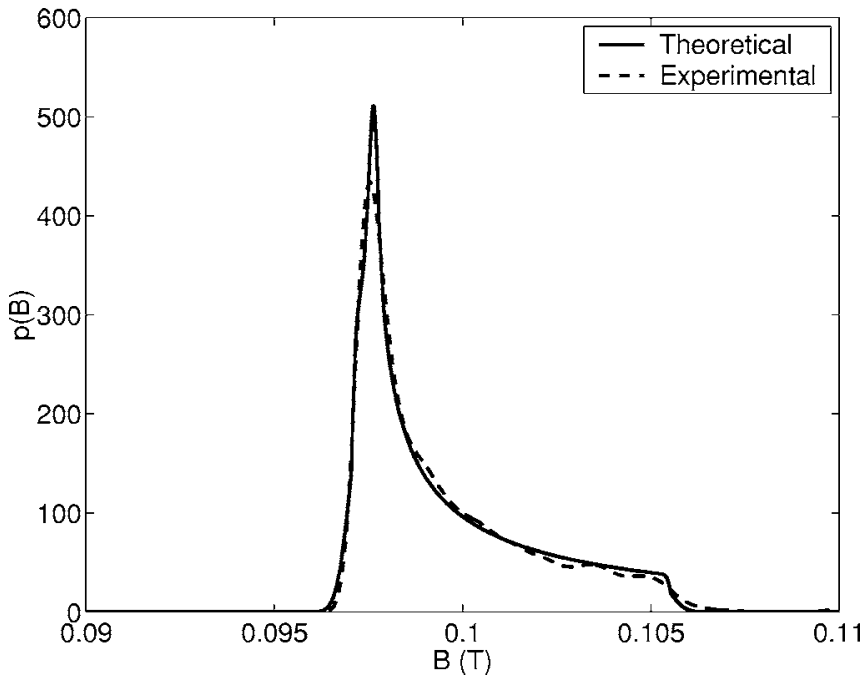

FIG. 8. $p(B)$ at zero current from Fig. 4 compared with a $p(B)$ from a solution to the GL equations. The latter has been smoothed by a Gaussian of rms width $3 \times 10^{-4} \mathrm{~T}$ to represent the finite resolution of the $\mu \mathrm{SR}$ technique. The $B(\boldsymbol{r})$ from the GL solution was used to generate the simulation in Fig. 9.

tity was also introduced by Delrieu ${ }^{25}$ ). For a typical set of experimental conditions, we can estimate the quantities in the simulation and compare its results with the experimental data. For an applied current of $15 \mathrm{~A}, V_{\mathrm{ff}}=0.21 \mathrm{mV}$, which corresponds to a vortex velocity of $v=0.14 \mathrm{~ms}^{-1}$. (Note that this voltage was determined from transport measurements at very low frequency, when the vortex lattice orientation is disordered by the sample edges. The vortex velocity may not have been quite the same under the higher-frequency conditions of the $\mu \mathrm{SR}$ measurements.) The spatial variation of the field $B(\boldsymbol{r})$ may be estimated by comparing the $0 \mathrm{~A}$ line shape of Fig. 4 to that from a solution to the Ginzburg-Landau (GL) equations (see Ref. 17 for more details). A good match was obtained from a solution with a GL parameter $\kappa$ of $\sim 4.0$ and $B / B_{c 2}$ of $\sim 0.36$; this is plotted in Fig. 8. The $p(B)$ for this field distribution has $\Delta B \approx 0.0085 \mathrm{~T}$. The equilibrium vortex line spacing corresponding to $B=0.1 \mathrm{~T}$ is $a_{0}=1.55$ $\times 10^{-7} \mathrm{~m}$. Hence, the $R$ expected from low frequency transport $\approx 0.78$.

Using a value of $R$, and the static vortex lattice $B(\boldsymbol{r})$ from the GL solution, a theoretical $p(B)$ for the moving lattice may be calculated. We found the best agreement between simulation and experiment with $R \approx 0.58$. This is shown in Fig. 9 (top) together with the corresponding experimentally determined $p(B)$ (bottom graph). The line shapes shown in Fig. 9 are in good agreement, and the difference in the $R$ value reflects the tendency seen in Fig. 7 for the flux flow voltage measured at low frequency to overestimate the flux flow speeds under ac conditions. The slight discrepancies between simulation and experiment may also be partly due to pinning (disorder tends to smear the van Hove singularities; see Ref. 26), the existence of a small range of orientations of the flowing vortex lattice (note that there is a trace of a central peak in the experimental data), and possibly a range of vortex velocities. It should also be noted that in order to obtain an explicit form for the $B(\boldsymbol{r})$ corresponding to the 

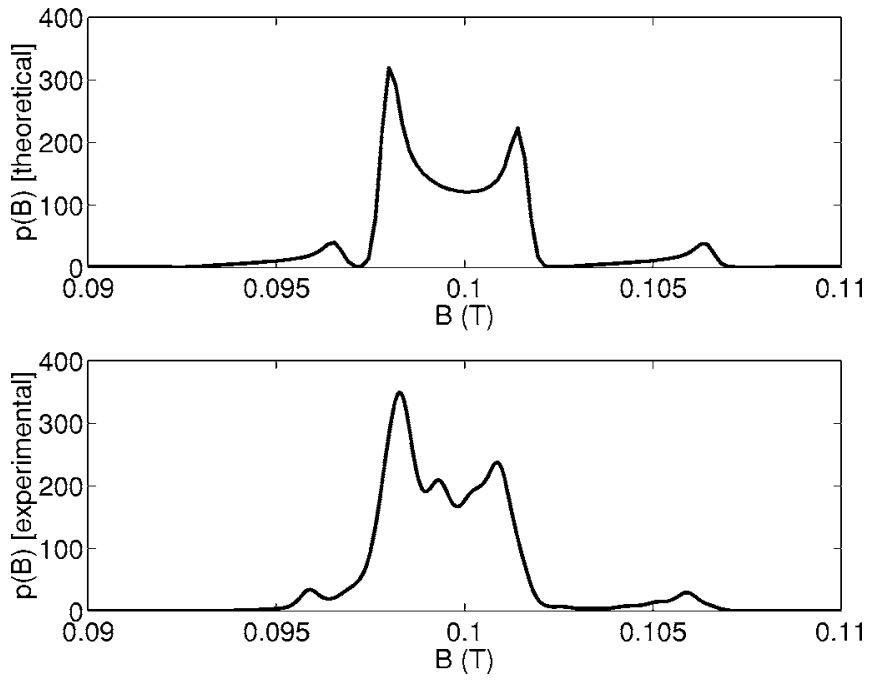

FIG. 9. The top $p(B)$ plot was calculated using the method developed by Ref. 17 for $B=0.1 \mathrm{~T}, R=0.58$, and no orientational disorder. The experimentally determined $p(B)$ (bottom) is identical to that shown in Fig. 4 for 15 A.

zero-current $p(B)$, we had to assume that the vortex lattice has the spatial variation of field given by a solution to the GL equations. With further development of both experiment and theory, it may be possible to obtain further information on these matters by detailed fitting of line shapes.

\section{CONCLUSIONS}

Our experimental results and calculations suggest that the interaction of vortex lines with the sample boundaries is the main factor determining the amount of disorder present in the moving vortex phase: for vortex lines which are not allowed to interact with the sample edges, the $\mu$ SR spectrum exhibits a characteristic double-peak structure, indicative of highly ordered motion along a principal vortex lattice vector. The speed of this motion is directly indicated by the position of "washboard frequency" sidebands in the spectrum. When vortices do cross the sample edges, motional narrowing of the $\mu \mathrm{SR}$ line shape is observed, which is characteristic of motion in which the vortex lattice is misaligned and probably disordered.

It is notable that the line shapes given by our results, and also in our modeling, contain many detailed features. It is likely that by further modeling we can gain more information about the vortex motion and the variation of field within the unit cell of the moving vortex lattice. This future effort will be aided by performing experiments on thin film samples which are either completely crystalline or completely amorphous (this will avoid possible disturbance of ordered vortex motion in our present polycrystalline samples). Thin films also have the advantage of a nearly two-dimensional instead of three-dimensional structure, which will be much closer to most of the simulations of vortex motion obtained so far. It is likely that the edges of thin films can be engineered to give a smoother entry for incoming vortices and hence reduce the edge effects that we observe. Thin film measurements will, however, require the use of low-energy muons, ${ }^{27}$ which can be implanted into a thin film, whereas the conventional highenergy muons that we have employed in this work require samples of $\sim 1 \mathrm{~mm}$ thickness.

\section{ACKNOWLEDGMENTS}

The experimental work was carried out at the Swiss Muon Source, Paul Scherrer Institut, Switzerland. We would like to thank E. H. Brandt for providing a copy of his code for solving the Ginzburg-Landau equations, and G. R. Walsh, D. Brewster, M. Parkes, J. Sutton, and A. Bradshaw for technical assistance. Fruitful discussions with M. W. Long, W. F. Vinen, R. Cywinski, C. E. Gough, and A. I. M. Rae are also acknowledged. D.C. was financially supported by the School of Physics \& Astronomy of the University of Birmingham, the A. G. Leventis Foundation, and the Committee of ViceChancellors and Principals.

\section{APPENDIX: ANALYTICAL DISCUSSION OF LINE SHAPES}

An analytical expression for the muon precession frequency distribution due to a moving vortex lattice may be obtained as follows. If the vortex lattice is moving as a whole with velocity $\boldsymbol{v}$, we may express the magnetic field distribution as the Fourier series ${ }^{25}$

$$
B(\boldsymbol{r})=\sum_{\boldsymbol{q} \in \mathcal{A}} B_{\boldsymbol{q}} e^{i \boldsymbol{q} \cdot(\boldsymbol{r}-\boldsymbol{v} t)},
$$

where $\mathcal{A}$ denotes the set of reciprocal lattice vectors $\boldsymbol{q}$ of the vortex lattice, and $B_{q}$ is the corresponding Fourier coefficient of the magnetic field distribution. Substitution of Eq. (A1) into Eq. (4) gives $P(\boldsymbol{r}, t)$ in the following form:

$$
\frac{P(\boldsymbol{r}, t)}{P(0)}=\exp \left\{i \gamma_{\mu} \int_{0}^{t} d t^{\prime}\left(B_{\mathbf{0}}+\sum_{\substack{\boldsymbol{q} \in \mathcal{A} \\ \boldsymbol{q} \neq \mathbf{0}}} B_{\boldsymbol{q}} \cos \left[\boldsymbol{q} \cdot\left(\boldsymbol{r}-\boldsymbol{v} t^{\prime}\right)\right]\right)\right\} \text {. }
$$

In the above, $B_{-q}=B_{q}$ and $B_{0}=\langle B\rangle$, the average magnetic induction. This expression can be used to calculate $p(B)$ by employing Eq. (3). We distinguish between two cases; first, when vortex motion does not occur along a Bragg plane, i.e., when $\boldsymbol{q} \cdot \boldsymbol{v} \neq 0$ for all $\boldsymbol{q} \in \mathcal{A}$, then integration of Eq. (A2) gives

$$
\frac{P(\boldsymbol{r}, t)}{P(0)}=f(\boldsymbol{r}) e^{i \gamma_{\mu}\langle B\rangle t} \prod_{\boldsymbol{q} \in \mathcal{A}^{*}} \exp \left\{i \frac{\gamma_{\mu} B_{q}}{\boldsymbol{q} \cdot \boldsymbol{v}} \sin [\boldsymbol{q} \cdot(\boldsymbol{r}-\boldsymbol{v} t)]\right\},
$$

where

$$
f(\boldsymbol{r})=\prod_{\boldsymbol{k} \in \mathcal{A}^{*}} \exp \left\{-i \frac{\gamma_{\mu} B_{\boldsymbol{k}}}{\boldsymbol{k} \cdot \boldsymbol{v}} \sin (\boldsymbol{k} \cdot \boldsymbol{r})\right\},
$$

and $\mathcal{A}^{*}=\{\boldsymbol{q}: \boldsymbol{q} \in \mathcal{A}, \boldsymbol{q} \cdot \boldsymbol{v} \neq 0, \boldsymbol{q} \neq \mathbf{0}\}$. Using the Bessel function expansion for the complex exponentials in Eq. (A3), we obtain 


$$
\frac{P(\boldsymbol{r}, t)}{P(0)}=f(\boldsymbol{r}) e^{i \gamma_{\mu}\langle B\rangle t} \prod_{\boldsymbol{q} \in \mathcal{A}^{*}}\left\{\sum_{n \in \mathbb{Z}} J_{n}\left(\frac{\gamma_{\mu} B_{q}}{\boldsymbol{q} \cdot \boldsymbol{v}}\right) e^{i n \boldsymbol{q} \cdot(\boldsymbol{r}-\boldsymbol{v} t)}\right\},
$$

where $J_{p}(z)$ denotes a Bessel function of the first kind, of order $p$ and argument $z$. The magnetic field probability distribution $p(\boldsymbol{r}, B)$, for a muon implanted at $\boldsymbol{r}$, is obtained by using

$$
p(\boldsymbol{r}, B)=\frac{\gamma_{\mu}}{2 \pi} \int_{-\infty}^{\infty} d t \frac{P(\boldsymbol{r}, t)}{P(0)} e^{-i \gamma_{\mu} B t},
$$

which gives

$$
\begin{aligned}
p(\boldsymbol{r}, B)= & f(\boldsymbol{r}) \sum_{\left\{n_{\boldsymbol{q}}\right\} \in \mathbb{Z}}\left\{\prod_{\boldsymbol{q} \in \mathcal{A}^{*}}\left[J_{n_{q}}\left(\frac{\gamma_{\mu} B_{\boldsymbol{q}}}{\boldsymbol{q} \cdot \boldsymbol{v}}\right)\right] \exp \left\{i \sum_{\boldsymbol{q} \in \mathcal{A}^{*}} n_{\boldsymbol{q}} \boldsymbol{q} \cdot \boldsymbol{r}\right\}\right. \\
& \left.\times \delta\left(B-\langle B\rangle+\frac{1}{\gamma_{\mu}} \sum_{\boldsymbol{q} \in \mathcal{A}^{*}} n_{\boldsymbol{q}} \boldsymbol{q} \cdot \boldsymbol{v}\right)\right\} .
\end{aligned}
$$

To calculate $p(B)$, we average $p(\boldsymbol{r}, B)$ over $\boldsymbol{r}$ within a vortex lattice unit cell. After further similar manipulation, we obtain

$$
p(B)=\left(\sum_{\left\{n_{q^{\prime}}\right\}} \prod_{\boldsymbol{q} \in \mathcal{A}^{*}}\left[J_{n_{q}}\left(\frac{\gamma_{\mu} B_{\boldsymbol{q}}}{\boldsymbol{q} \cdot \boldsymbol{v}}\right)\right]\right)^{2},
$$

where $\left\{n_{q}\right\}$ is a set of integers defined by

$$
\left\{n_{\boldsymbol{q}}\right\} \equiv\left\{n_{\boldsymbol{q}}: B=\langle B\rangle-\frac{1}{\gamma_{\mu}} \sum_{\boldsymbol{q} \in \mathcal{A}^{*}} n_{\boldsymbol{q}} \boldsymbol{q} \cdot \boldsymbol{v}\right\} .
$$

Delrieu $^{25}$ obtained a similar expression for the expected NMR line shape when the vortex velocity $\boldsymbol{v}$ satisfies $\boldsymbol{q} \cdot \boldsymbol{v}$ $\neq 0$ for all $\boldsymbol{q} \in \mathcal{A}$. However, his calculation relates to the square of the magnitude of the NMR line shape, even though the final result is similar to the one given above.

In the high velocity limit, the Bessel functions may be replaced by their asymptotic form for small argument, and the only term remaining is $p(B)=\delta(B-\langle B\rangle)$. This is the motional narrowing expected for the random or disordered direction of the vortex velocity.

Alternatively, when vortex motion takes place along a Bragg plane, then there exists a pair of reciprocal lattice vectors $\pm \boldsymbol{q}^{\prime} \in \mathcal{A}$ such that $\boldsymbol{q}^{\prime} \cdot \boldsymbol{v}=0$. In fact, there are infinitely many such vectors of the form $n \boldsymbol{q}^{\prime}$ (for integer $n$ ) which are orthogonal to $\boldsymbol{v}$, but we will only consider a firstorder $(n=1)$ reciprocal lattice vector for simplicity. In the limit $T \rightarrow T_{c}$ and $\langle B\rangle \geqslant 0.5 B_{c 2}$, this approximation is very good since $B_{q^{\prime}} \gg B_{n q^{\prime}}$. (This approximation becomes less good as $T \rightarrow 0$; in this case, the Fourier coefficients of the magnetic field decay much more slowly with $q$.) Assuming a single pair of reciprocal lattice vectors $\pm \boldsymbol{q}^{\prime}$ satisfying $\boldsymbol{q}^{\prime} \cdot \boldsymbol{v}$ $=0$, the muon polarization is given by

$$
\begin{aligned}
\frac{P(\boldsymbol{r}, t)}{P(0)}= & f(\boldsymbol{r}) \exp \left\{i \gamma_{\mu}\left[\langle B\rangle+B_{q^{\prime}} \cos \left(\boldsymbol{q}^{\prime} \cdot \boldsymbol{r}\right)\right] t\right\} \\
& \times \prod_{\boldsymbol{q} \in \mathcal{A}^{*}} \exp \left\{i \frac{\gamma_{\mu} B_{\boldsymbol{q}}}{\boldsymbol{q} \cdot \boldsymbol{v}} \sin [\boldsymbol{q} \cdot(\boldsymbol{r}-\boldsymbol{v} t)]\right\} .
\end{aligned}
$$

Proceeding in exactly the same way as before, it is easy to show that

$p(\boldsymbol{r}, B)$

$$
=\sum_{\left\{n_{q}\right\}} \sum_{\left\{m_{k}\right\}}\left\{\prod_{\boldsymbol{q} \in \mathcal{A}^{*}}\left[J_{n_{q}}\left(\frac{\gamma_{\mu} B_{q}}{\boldsymbol{q} \cdot \boldsymbol{v}}\right)\right] \prod_{\boldsymbol{k} \in \mathcal{A}^{*}}\left[J_{m_{k}}\left(\frac{\gamma_{\mu} B_{\boldsymbol{k}}}{\boldsymbol{k} \cdot \boldsymbol{v}}\right)\right] g(\boldsymbol{r})\right\},
$$

where

$$
g(\boldsymbol{r})=\exp \left\{i\left(\sum_{\boldsymbol{q} \in \mathcal{A}^{*}} n_{\boldsymbol{q}} \boldsymbol{q}-\sum_{\boldsymbol{k} \in \mathcal{A}^{*}} m_{\boldsymbol{k}} \boldsymbol{k}\right) \cdot \boldsymbol{r}\right\} \delta(h(\boldsymbol{r})),
$$

and

$$
h(\boldsymbol{r})=B-\langle B\rangle-B_{q^{\prime}} \cos \left(\boldsymbol{q}^{\prime} \cdot \boldsymbol{r}\right)+\frac{1}{\gamma_{\mu}} \sum_{\boldsymbol{q} \in \mathcal{A}^{*}} n_{q} \boldsymbol{q} \cdot \boldsymbol{v},
$$

and $m_{k}$ are integers defined in exactly the same way as $n_{q}$ in Eq. (A9). There are four values of $\boldsymbol{r}$ in the unit cell that satisfy $h(\boldsymbol{r})=0$ required by the $\delta$ function, and they give $g(\boldsymbol{r})$, which can then be averaged over the unit cell in Eq. (A11) to give

$$
\begin{aligned}
p(B)= & \sum_{\left\{m_{k}\right\}}\left\{\prod_{\boldsymbol{k} \in \mathcal{A}^{*}}\left[J_{m_{k}}\left(\frac{\gamma_{\mu} B_{\boldsymbol{k}}}{\boldsymbol{k} \cdot \boldsymbol{v}}\right)\right]\right\} \\
& \times \sum_{\left\{n_{q^{\prime}}\right\}}\left\{\prod_{\boldsymbol{q} \in \mathcal{A}^{*}}\left[J_{n_{q}}\left(\frac{\gamma_{\mu} B_{q}}{\boldsymbol{q} \cdot \boldsymbol{v}}\right)\right] \frac{1}{\pi\left|B_{q^{\prime}}\right|} \frac{1}{\sqrt{1-A_{q^{\prime}}^{2}}}\right\},
\end{aligned}
$$

where

$$
A_{\boldsymbol{q}^{\prime}} \equiv \frac{1}{B_{q^{\prime}}}\left(B-\langle B\rangle-\frac{1}{\gamma_{\mu}} \sum_{\boldsymbol{q} \in \mathcal{A}^{*}} n_{\boldsymbol{q}} \boldsymbol{q} \cdot \boldsymbol{v}\right) .
$$

In the limit $|\boldsymbol{v}| \rightarrow \infty, p(B)$ from Eq. (A8) tends to a $\delta$ function centered on the average field, $\langle B\rangle$. In the same limit for Eq. (A13), only terms with $n_{q}=0$ and $m_{k}=0$ contribute and $p(B)$ tends to the function

$$
p(B)=\frac{1}{\pi\left|B_{q^{\prime}}\right|} \frac{1}{\sqrt{1-\left(\frac{B-\langle B\rangle}{B_{q^{\prime}}}\right)^{2}}} .
$$

Equation (A15) gives a characteristic double-peak line shape, as shown in Fig. 6. In this simple approximation, $p(B)$ is symmetrical. If higher orders $n \boldsymbol{\boldsymbol { q } ^ { \prime }}$ are included, the highfield peak becomes weaker than the low-field peak.

In the more general case of finite velocity described by Eq. (A13), the line shape $p(B)$ consists of a rather compli- 
cated combination of line shapes of similar mathematical form to that of Eq. (A15). This combination of line shapes gives rise to the spectral sidebands, which look similar to the main $p(B)$ distribution but their shape appears to be more strongly affected by the high-field end of the main spectrum. This is due to the fact that the magnetic field varies more strongly around the vortex core. The position of the sidebands relative to the average field depends, in general, on the velocity of the vortex lines as well as on the direction of vortex motion relative to the vortex lattice unit cell. How- ever, the first sideband will appear, to a good approximation, at a magnetic field spacing from a main peak corresponding to the vortex lattice "washboard frequency."

Although the expressions obtained in this appendix are useful for obtaining explicit analytic forms for $p(B)$, they are cumbersome to use in calculation, due to the need to solve the conditions for the integers $m_{k}$ and $n_{q}$. It is for this reason that we have used the numerical method described in Ref. 17 to obtain field distributions from moving vortex lattices for comparison with experiment.
*Present address: Meteorological Service, Ministry of Agriculture, Natural Resources and Environment, 28 Nikis Avenue, 1086 Lefkosia, Cyprus. Electronic address: demetris.c@physics.org

'Present address: PR/PRL Editorial Offices, One Research Road, Box 9000, Ridge, NY 11961-9000.

Present address: Départment de Physique, Université de Fribourg, Ch. du Musée 3, CH-1700, Fribourg, Suisse.

${ }^{1}$ P. G. de Gennes, Superconductivity of Metals and Alloys (Addison-Wesley, Reading, MA, 1989).

${ }^{2}$ B. Josephson, Phys. Lett. 16, 242 (1965).

${ }^{3}$ P. Nozières and W. Vinen, Philos. Mag. 14, 667 (1966).

${ }^{4}$ J. Bardeen and M. Stephen, Phys. Rev. 140, A1197 (1965).

${ }^{5} \mathrm{M}$. Tinkham, Introduction to Superconductivity, 2nd ed. (McGraw-Hill, New York, 1996).

${ }^{6}$ P. Le Doussal and T. Giamarchi, Phys. Rev. B 57, 11356 (1998).

${ }^{7}$ S. Spencer and H. J. Jensen, Phys. Rev. B 55, 8473 (1997).

${ }^{8}$ T. Natterman and S. Scheidl, Adv. Phys. 49, 607 (2000).

${ }^{9}$ A. E. Koshelev and V. M. Vinokur, Phys. Rev. Lett. 73, 3580 (1994).

${ }^{10}$ C. Goupil et al., Physica C 341, 999 (2000).

${ }^{11}$ A. Pautrat, C. Goupil, C. Simon, D. Charalambous, E. M. Forgan, G. Lazard, P. Mathieu, and A. Brûlet, Phys. Rev. Lett. 90, 087002 (2003).
${ }^{12}$ E. M. Forgan, D. Charalambous, P. G. Kealey, P. J. C. King, R. Khasanov, and A. Amato, Physica B 326, 342 (2003).

${ }^{13}$ Z. L. Xiao, E. Y. Andrei, Y. Paltiel, E. Zeldov, P. Shuk, and M. Greenblatt, Phys. Rev. B 65, 094511 (2002).

${ }^{14}$ A. Campbell and J. Evetts, Adv. Phys. 21, 199 (1972).

${ }^{15}$ C. J. Olson, C. Reichhardt, and F. Nori, Phys. Rev. Lett. 81, 3757 (1998).

${ }^{16}$ E. M. Forgan, D. Charalambous, D. Fort, R. Cubitt, C. Simon, S. L. Lee, and A. Amato, J. Phys. IV 12, 155 (2002).

${ }^{17}$ D. Charalambous et al., Phys. Rev. B 66, 054506 (2002).

${ }^{18}$ A. Amato, GPS Users' Guide (Paul Scherrer Institut, Villigen, Switzerland, 2002), see also http://lmu.web.psi.ch

${ }^{19}$ B. Rainford and G. Daniell, Hyperfine Interact. 87, 1129 (1994).

${ }^{20}$ T. M. Riseman and E. M. Forgan, Physica B 326, 226 (2003).

${ }^{21}$ T. M. Riseman and E. M. Forgan, Physica B 326, 230 (2003).

${ }^{22}$ T. M. Riseman and E. M. Forgan, Physica B 326, 234 (2003).

${ }^{23}$ L. W. Couch, Digital and Analog Communications Systems, 6th ed. (Prentice Hall, New York, 2001), Chap. 5.

${ }^{24}$ A. Jánossy, C. Berthier, P. Ségransan, and P. Butaud, Phys. Rev. Lett. 59, 2348 (1987).

${ }^{25}$ J. M. Delrieu, J. Phys. F: Met. Phys. 3, 893 (1973).

${ }^{26}$ E. H. Brandt, J. Low Temp. Phys. 73, 355 (1988).

${ }^{27}$ E. Morenzoni et al., Physica B 326, 196 (2003). 